\title{
Vitamin D im Alter: Ist der Hype definitiv vorüber?
}

Autor

Heike A. Bischoff-Ferrari

Institut

Departement für Geriatrie und Altersforschung, Universitätsspital Zürich

Bibliografie

DOI https://doi.org/10.1055/a-0851-9737

Dtsch Med Wochenschr 2019; 144: 1018-1021

(c) Georg Thieme Verlag KG, Stuttgart · New York ISSN 0012-0472

\section{WAS IST NEU?}

Metaanalysen zur Wirkung von Vitamin D In den Jahren 2016 bis 2018 wurden 4 Metaanalysen publiziert, um den Nutzen von Vitamin D für die Frakturprävention zu untersu- chen. 2 dieser Metaanalysen konzentrierten sich auf die Primärprävention von Frakturen bei Erwachsenen ab 50 Jahren, bei denen kein Risiko für Frakturen und kein Vitamin-D-Mangel bestand. Eine weitere Metaanalyse konzentrierte sich auf die Kombination von Vitamin D plus Kalzium und die zuletzt publizierte Metaanalyse auf die individuelle Wirkung von Vitamin D ohne Kalzium. Bezogen auf die heutigen Richtlinien ist wichtig, dass sich nur 1 der 3 jüngsten Metaanalysen vorwiegend an Erwachsene ab 65 Jahren mit erhöhtem Risiko bezüglich Stürze, Frakturen und Vitamin-D-Mangel richtete.

Was können wir nun empfehlen? In Anbetracht und nach kritischer Prüfung der Metaanalysen zu Vitamin D und Frakturprävention ist eine Vitamin-D-Supplementation mit oder ohne Kalzium bei älteren Erwachsenen ab 65 Jahren mit einem erhöhten Risiko für Vitamin-D-Mangel und einem erhöhten Risiko für Frakturen weiterhin sinnvoll.

\section{Stand der Dinge}

Jüngste Metaanalysen zu Vitamin-D-Supplementation und Frakturreduktion haben Ärzte und Patienten dazu veranlasst, aktuelle Richtlinien zu Vitamin D infrage zu stellen. Grundsätzlich sind Vitamin D und Kalzium wichtige Nährstoffe für die Knochengesundheit; dazu ist ein Vitamin-D-Mangel ein etablierter Risikofaktor für Stürze, Knochenabbau und Frakturen bei über 65-jährigen. In den Jahren 2016 bis 2018 wurden 4 Metaanalysen publiziert, um den Nutzen von Vitamin D für die Frakturprävention zu untersuchen. Um die Ergebnisse dieser Analysen auf die klinische Versorgung zu übertragen, ist es wichtig, die Ziele und Zielgruppen sowie Einschränkungen dieser jüngsten Metaanalysen zu verstehen.

\section{Metaanalysen zur Wirkung von Vitamin D}

\section{Kombination von Vitamin D und Kalzium}

Die 2016 von Weaver et al. veröffentlichte Metaanalyse [1] hatte zum Ziel, die kombinierte Wirkung von Vitamin D mit Kalzium im Vergleich zu Placebo zu untersuchen. Diese Metaanalyse umfasst etwa $40 \%$ der qualitativ hochwertigen Daten zur Frakturreduktion mit Vitamin $D$, die zu den aktuellen Leitlinien mit einer Empfehlung von 800 IE Vitamin D beitrugen. Insgesamt fassten die Autoren 8 randomisiert-kontrollierte Studien zusammen ( $n=30970$ Erwachsene, überwiegendes Alter $65+$ ) und fanden
- eine signifikante $15 \%$ ige Reduktion aller Frakturen (RR $=0,85$; $95 \%-K I$ 0,73-0,98) und

- eine signifikante $30 \%$ ige Reduktion von Hüftfrakturen ( $R R=0,70,95 \%-K I ~ 0,56-0,87)$.

Die Kombination mit Kalzium führte dazu, dass Studien mit Bolusgaben von Vitamin D ausgeschlossen wurden und die meisten der eingeschlossenen Studien die heute empfohlene Dosis von 800 IE Vitamin D pro Tag untersuchten. Außerdem war der Großteil der Studienpopulation im Alter von 65 Jahren und älter und hatte damit ein erhöhtes Risiko für Stürze, Frakturen und Vitamin-DMangel; die Autoren schlossen zu Hause Lebende sowie Personen aus Institutionen ein, was den Anteil der Hochrisiko-Population für Vitamin-D-Mangel erhöhte.

\section{Klinische Relevanz}

Die Metaanalyse von Weaver et al. zeigte als einzige der 4 Metaanalysen eine signifikante Reduktion der Gesamtfrakturen und der Hüftfrakturen bei einer täglichen Kombination von Vitamin D plus Kalzium.

\section{Frakturprävention ab 50 Jahren}

2 der Metaanalysen konzentrierten sich auf die Primärprävention von Frakturen bei Erwachsenen ab 50 Jahren, bei denen kein Risiko für Frakturen und kein Vitamin-DMangel besteht [2, 3]. Die 2017 von Zhao et al. veröffentlichte Metaanalyse [2] hatte zum Ziel, die Wirkung von Vitamin D und Kalzium individuell sowie deren Kombination auf die Primärprävention von Frakturen bei zu Hause lebenden Erwachsenen ab 50 Jahren zu bewerten. Die 
Autoren schlossen 33 Studien mit insgesamt 51145 Männern und Frauen ein. Der primäre Endpunkt waren Hüftfrakturen; zu den sekundären Endpunkten gehörten jegliche nicht vertebrale Frakturen, vertebrale Frakturen und alle Frakturen. Die Autoren schlossen jede randomisierte klinische Studie mit einer Placebo-Kontrollgruppe oder ohne Behandlung in der Kontrollgruppe ein.

Die Autoren fanden keine signifikante Risikoreduktion von Vitamin D oder Kalzium bezogen auf das HüftbruchRisiko im Vergleich zu Placebo oder keiner Kontroll-Intervention:

- Vitamin D: RR: 1,21 (95\%-KI 0,99-1,47) und

- Kalzium: RR: 1,53 (95\%-KI, 0,97-2,42).

Weiterhin fanden die Autoren keine signifikante Risikoreduktion von kombiniertem Vitamin D plus Kalzium bezüglich Hüftbruch-Risiko (RR: 1,09 (95\%-KI 0,85$1,39)$ ). Auch wurde keine signifikante Verbesserung der anderen Fraktur-Endpunkte dokumentiert, und das weder für die individuellen Supplemente noch deren Kombination. Die Autoren führten zudem mehrere Untergruppenanalysen durch und dokumentierten, dass ihre Ergebnisse im Allgemeinen konsistent waren, unabhängig von Vitamin-D- oder Kalzium-Dosis, Geschlecht, vorbestehender Fraktur, Zufuhr von Kalzium aus der Ernährung und 25-Hydroxy-Vitamin-D-Blutspiegel.

Die Methodik und Konklusionen der Autoren wurden in einer publizierten Stellungnahme bezüglich verschiedener Punkte kritisiert [4]. So wählten die Autoren eine Zielgruppe aus, die weniger anfällig für eine niedrige Kalziumaufnahme und Vitamin-D-Mangel waren und ein geringes Frakturrisiko haben (Erwachsene ab 50 Jahren und Ausschluss älterer Erwachsener in Institutionen). Ferner wurden viele Studien eingeschlossen, die wenig Gelegenheit hatten, einen Nutzen der Interventionen zu demonstrieren. In einem Drittel der Studien war das Follow-Up zu kurz ( $\leq 12$ Monaten), um einen Nutzen für das Frakturrisiko durch die Interventionen nachzuweisen, da nicht sofort mit einem Nutzen für das Skelett gerechnet werden kann. Außerdem wurden 4 Studien mit einem qualitativ minderwertigen Studiendesign ohne Verblindung und ohne Behandlung in der Kontrollgruppe eingeschlossen. Und 1 Studie hatte einen inkorrekten Frakturbericht [5]. Ebenfalls wurde die Adhärenz bezüglich Studienmedikation nicht berücksichtigt, obgleich der Einfluss der Adhärenz in früheren Metaanalysen als signifikant belegt wurde [6, 7]. Bemerkenswerterweise nahmen in der stark gewichteten RECORD-Studie nur etwa die Hälfte der Teilnehmer ihre Vitamin-D- oder Kalziumpräparate ein [8]. 8 der 12 eingeschlossenen Studien zu Vitamin D gaben das Supplement in Bolusdosen (oral oder intramuskulär), was in der Literatur wiederholt Bedenken hinsichtlich der Förderung von Stürzen und Frakturen aufwirft [9, 10]. Insbesondere wurde verpasst, die Bolus-Studien in einer SubgruppenAnalyse separat auszuwerten.
Die US-Arbeitsgruppe für Präventivmaßnahmen (US Preventive Task Force) hat 2018 sorgfältig geprüft, ob und inwieweit ein Vitamin-D- oder Kalziumsupplement einzeln und in Kombination zur primären Vorbeugung von Frakturen bei Erwachsenen im Alter von über 50 Jahren beiträgt, für die kein Risiko für Osteoporose oder Vitamin-D-Mangel besteht [3, 11]. In Anbetracht der begrenzten Daten, die für die Primärprävention verfügbar sind, spricht sich das Gremium gegen eine tägliche Supplementation mit 400 IE oder weniger Vitamin D und 1000 mg oder weniger Kalzium zur Primärprävention von Frakturen bei Erwachsenen ohne Risiko aus. Für darüber liegende Vitamin-D- und Kalzium-Dosen lägen keine ausreichenden Nachweise vor, um einen Nutzen zu bewerten. Das Gremium identifizierte beispielsweise eine große Studie mit 4-monatlichen Gaben von 100000 IE Vitamin D mit einem signifikanten Nutzen für das Frakturrisiko [12] und eine große Studie mit monatlichen 100000 IE Vitamin D ohne ein Nutzen für das Frakturrisiko [13].

\section{Klinische Relevanz}

Bei den Metaanalysen von Zhao et al. [2] und der US Preventive Task Force [3] geben beide Autorenteams an, dass ihre Empfehlungen nur für gesunde, zu Hause lebende Erwachsene im Alter ab 50 Jahren gelten, bei denen keine Osteoporose und kein Vitamin-D-Mangel bekannt sind. Ihre Ergebnisse beziehen sich daher nicht auf das große Segment älterer erwachsener Menschen mit einem erhöhten Risiko für Knochenbrüche und Vitamin-D-Mangel, bei denen die Literatur günstige Wirkungen inklusive Sturzprävention belegen konnte [11].

\section{Individuelle Wirkung von Vitamin D}

Die zuletzt publizierte Metaanalyse untersuchte die individuelle Wirkung von Vitamin D ohne Kalzium [14]. Die Autoren identifizierten 81 verblindete und nicht verblindete randomisierte Studien mit insgesamt 44790 Erwachsenen über 50 Jahren. In den berücksichtigten Studien wurde Vitamin D mit Placebo oder unbehandelte Kontrollen, oder Vitamin D mit einer anderen Dosierung verglichen. Die Autoren berichten über keinen Effekt der Vitamin-D-Supplementation auf Frakturen und Stürze. Sie berichten auch über keinen Nutzen von Vitamin D für die Knochenmineraldichte (BMD), obgleich bei 3 von 5 Knochendichte-Messorten der Nutzen von Vitamin D signifikant war:

- $0,34 \%$ auf die gesamte Hüft-Knochendichte $(p=0,002)$,

- $0,76 \%$ auf den Schenkelhals $(p<0,001)$ und

- $0,25 \%$ auf die Lendenwirbelsäule $(p=0,05)$.

Das deutet darauf hin, dass Vitamin D tatsächlich einen, wenn auch kleinen, Nutzen für die Knochendichte hat, und das insbesondere an der Hüfte. 
In einer publizierten Kritik und Re-Analyse dieser Metaanalyse wurden mehrere weitere Einschränkungen in der Methodik und Interpretation dieser Arbeit diskutiert [15]:

1. Die Autoren schlossen einen wesentlichen Teil der Literatur zu Vitamin D aus, nämlich alle Studien, in denen Vitamin D mit Kalzium kombiniert und mit Placebo verglichen wurde. Solche Studien machen etwa $40 \%$ der qualitativ hochwertigen Daten zur Frakturreduktion aus und haben zu den aktuellen Leitlinien mit einer Empfehlung von 800 IE Vitamin D beigetragen. Das Ausmaß dieser Verzerrung wird in der Metaanalyse von 2016 von Weaver et al. beschrieben (siehe oben), die eine signifikante Reduktion der gesamten Frakturen um $15 \%$ und eine Reduktion der Hüftfrakturen um $30 \%$ dokumentierte [1].

2. Die Autoren schlagen vor, die aktuellen Empfehlungen zu Vitamin D entsprechend ihren Ergebnissen zu überarbeiten. Aktuelle Richtlinien beziehen sich jedoch auf eine tägliche Dosis von 800 IE bis 1000 IE Vitamin D, während niedrigere Dosen als unwirksam angesehen werden [16, 17]. Außerdem ist gut dokumentiert, dass große jährliche Bolus-Applikationen von Vitamin D nicht mehr empfohlen werden aufgrund eines mehrfach dokumentierten erhöhten Sturz- und Frakturrisikos $[10,18]$. In einer publizierten Re-Analyse von 8 randomisierten, Placebo-kontrollierten Studien der Autoren bezüglich Gesamtfrakturen und 11 Placebokontrollierten Studien der Autoren bezüglich Stürze, die 800 bis 1000 IE Vitamin D mit mehr als 50 \% Adhärenz testeten ohne Berücksichtigung der großen jährlichen Dosierungsversuche, zeigte sich eine signifikante Reduktion der Gesamtfrakturen um 14\% ( $R R=0,86$; $95 \%-K I 0,75-0,98)$ und eine signifikante Verringerung der Stürze um $12 \%$ (RR=0,88; $95 \%-K I$ 0,81-0,95).

Klinische Relevanz

Zusammenfassend sind die Limitierungen dieser Metaanalyse wesentlich und es ist zu hinterfragen, inwieweit Folgerungen für die klinische Versorgung ableitbar sind. Tatsächlich lässt sich aus der oben beschriebenen Re-Analyse der Richtlinien-relevanten Studien ein gegenteiliges Signal ablesen, nämlich dass eine VitaminD-Supplementation in einer täglichen Dosis von 800 bis 1000 IE das Sturz- und Frakturrisiko verringert.

\section{Was können wir nun empfehlen?}

Basierend auf den Einschränkungen der Bolland-Metaanalyse [14] und der Zielgruppe von Zhao et al. [2] und der US Preventive Task Force [3] von Erwachsenen ohne Osteoporose und Vitamin-D-Mangel ist es möglicherweise zu früh, anhand dieser neuen Metaanalysen eine Aufhebung der Empfehlungen zu Vitamin D mit oder ohne Kalzium zur Vorbeugung von Frakturen bei allen älteren Erwachsenen anzuregen. Insbesondere für ältere Erwachsene mit einem erhöhten Risiko für Frakturen und/oder
Vitamin-D-Mangel ist es weiterhin sinnvoll, 800 bis 1000 IE Vitamin D pro Tag einzunehmen, analog den Empfehlungen der International Osteoporosis Foundation [19], der US Endocrine Society [20] und NOF [21].

Hingegen sind anhand der heutigen Studienlage große jährliche Bolus-Applikationen von Vitamin D (300 000 bis $500000 \mathrm{IU}$ ) bei älteren Erwachsenen mit einem Risiko für Frakturen schädlich und sollten in der klinischen Versorgung nicht fortgesetzt werden. Bei einer größeren monatlichen Dosis von 100000 IE ist eine weitere Bewertung in Bezug auf Wirksamkeit und Sicherheit erforderlich.

Die Reduzierung des Frakturrisikos bei gebrechlichen älteren Erwachsenen ab 65 Jahren, die 75 \% aller osteoporotischen Frakturen aufweisen [22], bleibt ein wichtiges Ziel für die Volksgesundheit.

\section{Klinische Relevanz \\ Angesichts des geringen Risikos und der geringen Kosten von Vitamin D halten wir es für wichtig, ältere Erwach- sene nicht von der täglichen Einnahme von 800 bis 1000 IE Vitamin D abzuhalten und die optimale Dosis und Anwendung von Vitamin D weiter zu untersuchen.}

\section{Interessenkonflikt}

HBF erhielt Vortragshonorare von Sandoz, Wild, Sanofi, Roche-Diagnostics, Meda und Vifor. HBF erhielt Forschungsgelder im Bereich Vitamin D und Muskelgesundheit von DSM Nutritional Products, sowie unabhängige Forschungsgelder von Pfizer, Vifor und Wild.

Autorinnen/Autoren

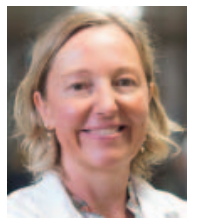

Prof. Dr. med. Heike A. Bischoff-Ferrari

ist Klinikdirektorin an der Klinik für Geriatrie des Universitätsspitals Zürich und Leiterin des Zentrums Alter und Mobilität, Universitätsspital Zürich und Stadtspital Waid.

Heike.Bischoff@usz.ch

Korrespondenzadresse

Prof. Dr. med. Heike A. Bischoff-Ferrari, DrPH

Klinik für Geriatrie, Universitätsspital Zürich

Rämistrasse 100

8091 Zürich

Heike.Bischoff@usz.ch

Literatur

[1] Weaver CM, Alexander DD, Boushey C] et al. Calcium plus vitamin $\mathrm{D}$ supplementation and risk of fractures: an updated meta-analysis from the National Osteoporosis Foundation. Osteoporos Int 2016; 27: 367-376 
[2] Zhao JG, Zeng XT, Wang J et al. Association Between calcium or Vitamin D Supplementation and Fracture Incidence in Community-Dwelling Older Adults: A Systematic Review and Meta-analysis. JAMA 2017; 318: 2466-2482

[3] US Preventive Services Task Force et al. Vitamin D, calcium, or Combined Supplementation for the Primary Prevention of Fractures in Community-Dwelling Adults: US Preventive Services Task Force Recommendation Statement. JAMA 2018; 319: 1592-1599

[4] Bischoff-Ferrari HA, Dawson-Hughes B, Willett WC. Issues of trial selection and subgroup considerations in the recent meta-analysis of Zhao and colleagues on fracture reduction by Calcium and vitamin D supplementation in community-dwelling older adults. Osteoporos Int 2018; 29: 2151-2152

[5] Bischoff-Ferrari HA, Rees JR, Grau MV et al. Effect of calcium supplementation on fracture risk: a double-blind randomized controlled trial. Am J Clin Nutr 2008; 87: 1945-1951

[6] Bischoff-Ferrari HA, Willett WC, Wong JB et al. Prevention of nonvertebral fractures with oral vitamin $D$ and dose dependency: a meta-analysis of randomized controlled trials. Arch Intern Med 2009; 169: 551-561

[7] Bischoff-Ferrari HA, Willett WC, Orav E] et al. A pooled analysis of vitamin D dose requirements for fracture prevention. N Engl J Med 2012; 367: 40-49

[8] Grant AM, Avenell A, Campbell MK et al. Oral vitamin D3 and Kalzium for secondary prevention of low-trauma fractures in elderly people (Randomised Evaluation of Kalzium Or vitamin D, RECORD): a randomised placebo-controlled trial. Lancet 2005; 365: 1621-1628

[9] Bischoff-Ferrari HA, Dawson-Hughes B, Platz A et al. Effect of high-dosage cholecalciferol and extended physiotherapy on complications after hip fracture: a randomized controlled trial. Arch Intern Med 2010; 170: 813-820

[10] Sanders KM, Stuart AL, Williamson E] et al. Annual high-dose oral vitamin $\mathrm{D}$ and falls and fractures in older women: a randomized controlled trial. JAMA 2010; 303: 1815-1822

[11] Bischoff-Ferrari HA, Bhasin S, Manson JE. Preventing Fractures and Falls: A Limited Role for Kalzium and Vitamin D Supplements? JAMA 2018; 319: 1552-1553

[12] Trivedi DP, Doll R, Khaw KT. Effect of four monthly oral vitamin D3 (cholecalciferol) supplementation on fractures and mortality in men and women living in the community: randomised double blind controlled trial. BMJ 2003; 326: 469
[13] Khaw KT, Stewart AW, Waayer D et al. Effect of monthly highdose vitamin D supplementation on falls and non-vertebral fractures: secondary and post-hoc outcomes from the randomised, double-blind, placebo-controlled ViDA trial. Lancet Diabetes Endocrinol 2017; 5: 438-447

[14] Bolland MJ, Grey A, Avenell A. Effects of vitamin D supplementation on musculoskeletal health: a systematic review, meta-analysis, and trial sequential analysis. Lancet Diabetes Endocrinol 2018; 6: 847-858

[15] Bischoff-Ferrari HA, Orav EJ, Abderhalden L et al. Vitamin D supplementation and musculoskeletal health. Lancet Diabetes Endocrinol 2019; 7: 85

[16] Bischoff-Ferrari HA, Willett WV, Orav E] et al. A pooled analysis of vitamin $\mathrm{D}$ dose requirements for fracture prevention. N Engl J Med 2012; 367: 40-49

[17] International Osteoporosis Foundation. Guideline on Vitamin D. Im Internt: https://www.iofbonehealth.org/osteoporosismusculoskeletal-disorders/osteoporosis/prevention/vitamind. Letzter Zugriff: 19.6.2019

[18] Smith H, Anderson F, Raphael H et al. Effect of annual intramuscular vitamin $\mathrm{D}$ on fracture risk in elderly men and women-a population-based, randomized, double-blind, placebo-controlled trial. Rheumatology (Oxford) 2007; 46: 1852-1857

[19] Dawson-Hughes B, Mithal A, Bonjour JP et al. IOF position statement: vitamin $\mathrm{D}$ recommendations for older adults. Osteoporos Int 2010; 21: 1151-1154

[20] Holick MF, Binkley NC, Bischoff-Ferrari HA et al. Evaluation, treatment, and prevention of vitamin D deficiency: an Endocrine Society clinical practice guideline. J Clin Endocrinol Metab 2011; 96: 1911-1930

[21] Cosman F, de Beur SJ, LeBoff MS et al. Clinician's Guide to Prevention and Treatment of Osteoporosis. Osteoporos Int 2014; 25: 2359-2381

[22] Melton L], Crowson CS, O'Fallon WM. Fracture incidence in Olmsted County, Minnesota: comparison of urban with rural rates and changes in urban rates over time. Osteoporos Int 1999; 9: 29-37 